\title{
CHẤT LƯợNG DỊCH VỤ GIÁO DỤC ĐẠI HỌC THEO MÔ HİNH IPA TẠI TRƯÒ̀NG ĐẠI HỌC TÀI CHÍNH - MARKETING
}

\section{ASSESSMENT OF HIGHER EDUCATION SERVICE QUALITY UNDER THE IPA MODEL AT THE UNIVERSITY OF FINANCE - MARKETING}

\author{
Bảo Trung, Nguyễn Thị Tố Loan ${ }^{1}$
}

Ngày nhận bài: 17/02/2020 Ngày chấp nhận đăng: 10/3/2020 Ngày đăng: 05/06/2020

\section{Tóm tắt}

Bài báo xác định các thành phần và phân tích chất lượng dịch vụ giáo dục đại học theo mô hình Important - Performance Analysis (IPA - Phân tích Tầm quan trọng - Việc thực hiện) tại Trường Đại học Tài chính - Marketing. Nghiên cứu định tính được thực hiện thông qua thảo luận nhóm 10 người xác định 5 thành phần với 25 biến quan sát chất lượng dịch vụ giáo dục đại học dưới góc nhìn sinh viên. Nghiên cứu định lượng được thực hiện thông qua phỏng vấn hai lần với 193 mẫu. Phỏng vấn lần 1 đo lường mức độ quan trọng và phỏng vấn lần 2 đo lường mức độ thực hiện. Dữ liệu khảo sát được xử lý thông qua thống kê mô tả, đo lường độ tin cậy thang đo Cronbach Alpha, kiểm định Paired Sample T-test đối với P-I. Kết quả nghiên cứu đo lường chất lượng dịch vụ giáo dục đại học theo mô hình IPA tại trường Đại học Tài chính - Marketing gồm Phần I - Tập trung phát triển có 5 các biến quan sát; Phần II - Tiếp tục duy trì, 12 biến quan sát; Phần III - U’u tiên thấp, 8 biến quan sát; Phần IV - Hạn chế đầu tư không có biến quan sát nào. Trên cơ sở đánh giá chất lượng dịch vụ giáo dục đại học theo mô hình IPA, bài báo đề xuất một số hàm ý quản trị.

Tù khóa: Chất lượng dịch vụ, Chất lượng dịch vụ giáo dục đại học, mô hình IPA, Trường Đại học Tài chính - Marketing.

\begin{abstract}
The paper identifies the components and measure the service quality in higher education based on the Important - Performance Analysis model (IPA) at the University of Finance - Marketing. Qualitative research was conducted through group discussion of 10 people identifying 5 components with 25 observed variables of the higher education service quality from the perspective of students. Quantitative research was conducted through double interviews with 193 samples. The 1 st interview measures the importance level and the 2nd interview measures the level of performance. Survey data is processed through descriptive statistics, Cronbach Alpha scale reliability measurement, Paired Sample T - test for P-I. Research results on measuring service quality under the IPA model at the
\end{abstract}

${ }^{1}$ Trường Đại học Tài chính - Marketing 
University of Finance - Marketing include Part I - Concentrate here has 5 observed variables; Part II - Keep up the Good Work, 12 observed variables; Part III - Low priority, 8 observed variables; Part IV - Possible Overskill with no observed variables. Based on the assessment of the quality of higher education services under the IPA model, the paper proposes some managerial implications.

Keywords: Service quality, Service quality in higher education, IPA, University of Finance Marketing.

\section{Giới thiệu}

Chất lượng dịch vụ giáo dục đại học luôn luôn là mối quan tâm hàng đầu của nhiều đối tượng dù có tham gia hoặc không tham gia vào quá trình giáo dục. Khi các trường đại học tại Việt Nam ra đời ngày càng nhiều và việc cạnh tranh về đào tạo rất lớn giữa các trường đại học công lập và ngoài công lập; giữa các trường đại học Việt Nam và đại học nước ngoài thì việc nâng cao chất lượng dịch vụ giáo dục đại học là một yêu cầu bức thiết hiện nay đối với các trường đại học nhằm tăng sức cạnh tranh. Mặc dù, chất lượng dịch vụ giáo dục đại học dưới góc nhìn sinh viên đã được nghiên cứu nhiều trong và ngoài nước nhưng do văn hóa khác nhau, sinh viên ở mỗi quốc gia có thể cảm nhận khác nhau về chất lượng dịch vụ giáo dục đại học. Do đó, các thành phần chất lượng dịch vụ giáo dục đại học có khác nhau.

Hiện nay cũng có nhiều mô hình nghiên cứu về chất lượng giáo dục đại học liên quan. Ford, J.B, Mathew, Joseph. Ford, JR và cộng sự (1999) thực hiện "Phân tích mức độ thực hiện - tầm quan trọng (IPA) như là công cụ chiến lược cho thị trường dịch vụ: Nghiên cứu trường hợp cảm nhận chất lượng dịch vụ cho sinh viên ngành kinh doanh ở New Zealand và USA". Joseph và Joseph (1997) nghiên cứu "Chất lượng dịch vụ giáo dục: một góc nhìn sinh viên”. (Huỳnh Văn Thái, 2017), nghiên cứu "Vận dụng mô hình IPA để đánh giá chất lượng dịch vụ đào tạo tại Trường Cao đẳng Công nghiệp Tuy Hòa”. Mặc dù đã có một số nghiên cứu có liên quan đến mô hình IPA nhưng do thành phần chất lượng dịch vụ giáo dục đại học còn nhiều quan điểm khác nhau và các nghiên cứu cũng không thống nhất.

Nghiên cứu này xác định các thành phần chất lượng dịch vụ giáo dục dưới góc nhìn sinh viên trường Đại học Tài chính - Marketing và từ đó sử dụng mô hình IPA đánh giá và đề xuất một số hàm ý quản trị cho Trường.

\section{Cơ sở lý thuyết và mô hình nghiên cứu}

\subsection{Chất lự̣ng dịch vụ giáo dục đại học}

Chất lượng dịch vụ là một khái niệm được quan tâm và tranh luận trong nhiều tài liệu nghiên cứu bởi vì những khó khăn về định nghĩa và đo lường chất lượng dịch vụ không có sự đồng thuận chung. Hiện nay có nhiều quan điểm khác nhau của các nhà nghiên cứu hàn lâm trên thế giới về định nghĩa chất lượng dịch vụ nhưng mô hình đang được nhiều học giả quan tâm là mô hình của Parasuraman và các cộng sự là những người tiên phong về lĩnh vực này. Parasuraman và cộng sự (1985) định nghĩa chất lượng dịch vụ là mức độ khác nhau giữa sự mong đợi của người tiêu dùng về dịch vụ và nhận thức của họ về kết quả của dịch vụ (Parasuraman, 1985).

Các nhà nghiên cứu hàn lâm trên thế giới đưa ra những quan điểm khác nhau về chất lượng dịch vụ giáo dục đại học. Harvey và Green cho rằng chất lượng dịch vụ giáo dục đại học có thể tập hợp thành 5 nhóm quan điểm về chất lượng như sau: (1) Chất lượng là sự vượt trội; (2) Chất lượng là sự hoàn hảo; (3) Chất lượng là phù hợp với mục tiêu; (4) Chất lượng là giá trị đồng tiền; 
(5) Chất lượng là sự chuyển đổi (Harvey, L., and Green, D., 1993). Cheng và Tam đề nghị rằng chất lượng giáo dục đại học là tập hợp các yếu tố đầu vào, quy trình và đầu ra của hệ thống đào tạo cung cấp dịch vụ hoàn toàn thỏa mãn cả những khách hàng chiến lược bên trong và bên ngoài bằng cách đáp ứng những mong đợi hiện tại và tiềm ẩn của họ (Cheng, Y.C and Tam, W.M., 1997). O'Neil và Palmer cho rằng chất lượng giáo dục đại học là sự khác biệt giữa những gì mà sinh viên mong muốn nhận được và cảm nhận của họ về chuyển giao thực tế (O’Neil and Palmer, 2004).

Như vậy, chất lượng giáo dục đại học là một khái niệm phức tạp và đa hướng và một định nghĩa phù hợp duy nhất về chất lượng giáo dục đại học vẫn còn thiếu (Harvey và Green, 1993). Trong nghiên cứu này, định nghĩa chất lượng giáo dục đại học của O’Neil và Palmer (2004) được sử dụng vì nó liên quan đến cảm nhận của sinh viên về chất lượng dịch vụ giáo dục đại học.

\subsection{Thành phần chất lự̛ng dịch vụ giáo duc dại học}

\subsubsection{Chương trình đào tạo}

Có rất nhiều quan niệm khác nhau về chương trình đào tạo, mỗi cách tiếp cận sẽ có định nghĩa khác nhau về chương trình đào tạo. Chương trình đào tạo là cung cấp các cơ hội học tập theo kế hoạch của cơ sở giáo dục cho người học và những trải nghiệm mà người học gặp phải khi chương trình đào tạo được thực hiện (Print, 1987). NAAC (2008) cho rằng chương trình đào tạo là thành phần thiết yếu của bất kỳ hệ thống đào tạo không phân biệt mức độ đào tạo. Tất cả phương diện khác như giảng dạy, học tập và đánh giá hoặc nghiên cứu và phát triển cơ sở hạ tầng và nguồn lực học tập, các hoạt động của sinh viên và hệ thống hỗ trợ đều xoay quanh nó (NAAC, 2008). Nội dung của chương trình đào tạo có mục tiêu rõ ràng, có chuẩn đầu ra rõ ràng, có kiến thức kỹ năng đáp ứng nhu cầu thực tế, nội dung chương trình đào tạo giúp sinh viên tìm công việc phù hợp. Chính chương trình đào tạo tốt sẽ mang đến những chất lượng dịch vụ giáo dục đại học tốt đáp ứng mục tiêu giáo dục đề ra (Joseph, M. and Joseph, B., 1997); (Firdaus, 2006); (Tsinidou, M \& et al., 2010); (Vijaya Sunder, 2015); (Silva \& et al., 2017).

\subsubsection{Kỹy năng giảng dạy của giảng viên}

Kỹ năng giảng dạy của giảng viên là những hoạt động riêng rẽ và gắn kết của giảng viên mà thúc đẩy sinh viên học (Kyriacou, 2007). Kyriacou (2007) cho rằng 3 yếu tố quan trọng của kỹ năng giảng dạy của giảng viên có thể nhận thấy là: (1) Kiến thức bao gồm kiến thức của giảng viên về môn học, sinh viên, chương trình giảng dạy, phương pháp giảng dạy; (2) Ra quyết định bao gồm những suy nghĩ và ra quyết định xảy ra trước, trong và sau một bài giảng, (3) Hành động bao gồm những hành động của giảng viên thực hiện để thúc đẩy học tập sinh viên. Kỹ năng giảng dạy là những chiến lược mà giảng viên sử dụng để tạo thuận lợi cho việc học của sinh viên và được công nhận bởi những người có thẩm quyền để đánh giá như là kỹ năng (Wragg, 2005). Các nghiên cứu trước đều chỉ ra kỹ năng giảng dạy của giảng viên là thành phần quan trọng của chất lượng dịch vụ giáo dục đại học (Silva \& et al., 2017); (Vijaya Sunder, 2015).

\subsubsection{Co sở vật chất}

Cơ sở vật chất là tất cả phương tiện vật chất được huy động vào việc giảng dạy, học tập và các hoạt động mang tính giáo dục khác để đạt được mục đích giáo dục. Cơ sở vật chất đầy đủ và hiện đại như thư viện và các thiết bị để sử dụng, kể cả công nghệ thông tin, sách giáo khoa, môi trường học tập và sinh hoạt, và tất cả trang thiết bị mà hỗ trợ giảng dạy của giảng viên và sinh viên và quy trình học tập là yếu tố quan trọng tạo nên chất lượng giáo dục đại học (Silva \& et al., 2017); (Vijaya Sunder, 2015); (Jain, 2013); (Firdaus, 2006). 
2.2.4. Tương tác giữa nhà trương và doanh nghiệp

Tương tác/quan hệ hợp tác giữa nhà trường và doanh nghiệp được hiểu là tất cả mọi hình thức tương tác trực tiếp hay gián tiếp, có tính chất cá nhân hay tổ chức giữa trường đại học và các doanh nghiệp nhằm hỗ trợ lẫn nhau vì lợi ích của cả hai: hợp tác trong nghiên cứu và phát triển, kích thích sự vận động năng động qua lại của giảng viên, sinh viên và các nhà chuyên môn đang làm việc tại các doanh nghiệp; thương mại hóa các kết quả nghiên cứu; xây dựng chương trình đào tạo; tổ chức học tập suốt đời; hỗ trợ các nỗ lực sáng nghiệp và quản trị tổ chức (Phạm Thị Ly, 2013). Thành công của mối quan hệ hợp tác này dẫn đến kết quả nâng cao chất lượng dịch vụ giáo dục đại học và triển vọng việc làm tương lai cho sinh viên, hay những nghiên cứu do các trường thực hiện và việc chuyển giao công nghệ, tri thức cho khu vực sản xuất kinh doanh (Lagrosen, 2004); (Jain, 2013); (Phạm Thị Ly, 2013).

\subsubsection{Hoạt động ngoại khóa}

Hoạt động ngoại khóa là một hình thức tổ chức dạy học, là một dạng hoạt động của sinh viên tiến hành ngoài giờ lên lớp chính thức, ngoài phạm vi quy định của chương trình bộ môn nhằm hỗ trợ cho chương trình nội khóa, góp phần hoàn thiện và phát triển nhân cách, bồi dưỡng năng khiếu và tài năng sáng tạo của sinh viên, giúp sinh viên không chỉ củng cố và khắc sâu kiến thức, rèn luyện kỹ năng bồi dưỡng tình cảm mà còn tạo ra sự thoải mái, hòa nhập với tập thể, nâng cao ý thức cuộc sống cộng đồng, đáp ứng tâm lý ưa sôi động, thích khám phá cái mới của tuổi trẻ. Các nghiên cứu thực nghiệm đã chỉ ra hoạt động ngoại khóa là thành phần quan trọng của chất lượng dịch vụ giáo dục đại học (Peng, P.J. and Samah, A.J.A, 2006); (Sumaedi, 2012).

\subsubsection{Chất lượng dịch vụ hỗ trợ sinh viên.}

Chất lượng dịch vụ hỗ trợ sinh viên là chất lượng các dịch vụ hỗ trợ như các hướng dẫn, hỗ trợ kỹ thuật, tiếp cận thư viện và dịch vụ thông tin, tư vấn,... phải phù hợp và có giá trị với sinh viên (Simpson, 2002). Chất lượng dịch vụ hỗ trợ sinh viên là đề cập đến một loạt các chất lượng tương tác phi học thuật giữa sinh viên với trường đại học. Nó bao gồm trước tuyển sinh, tư vấn học tập, đăng ký, định hướng, tư vấn nghề nghiệp, dịch vụ thư viện, nhà sách, tư vấn hỗ trợ tài chính, tư vấn cá nhân, hỗ trợ xã hội, hỗ trợ kỹ thuật, lập kế hoạch chương trình, kiểm tra bằng cấp và sinh viên tốt nghiệp, và đánh giá bảng điểm (Dirr, 2014). Các nghiên cứu trước cũng chỉ ra chất lượng dịch vụ hỗ trợ sinh viên là thành phần quan trọng của chất lượng dịch vụ giáo dục đại học (Peng, P.J. and Samah, A.J.A, 2006); (Firdaus, 2006); (Jain, 2013).

\subsection{Mô hình nghiên cứu chất lự̛ng dịch vu giáo dục đại hoc theo IPA}

Mô hình phân tích mức độ quan trọng và thực hiện dịch vụ Importance Performance Analysis do Martilla và James xây dựng vào năm 1977. Tầm quan trọng (Important) là chất lượng của nó là đáng kể, có giá trị, hoặc cần thiết trong một tình huống cụ thể. Tầm quan trọng có nghĩa là có ảnh hưởng, sức mạnh hoặc trạng thái. Việc thực hiện (Performance) là làm cho trở thành sự thật bằng những việc làm hoặc hành động cụ thể. IPA là mô hình đo lường chất lượng dịch vụ dựa vào sự khác biệt giữa ý kiến khách hàng về mức độ quan trọng của các chỉ tiêu mà khách hàng đánh giá (I-P gaps). Mô hình Importance Performance Analysis (IPA) được thực hiện bằng cách so sánh hai tiêu chuẩn hình thành nên quyết định lựa chọn của khách hàng, cụ thể: (1) Tầm quan trọng tương đối của các thuộc tính chất lượng, và (2) Mức độ thực hiện của các thuộc tính chất lượng. Kết quả của sự phân tích mức độ quan trọng và mức độ thực hiện được thực hiện lên sơ đồ IPA với trục tung $(\mathrm{Y})$ thể hiện mức độ thực hiện và trục hoành $(\mathrm{X})$ thể hiện mức độ quan trọng (Martilla J.A. \& James J.C. , 1977). 


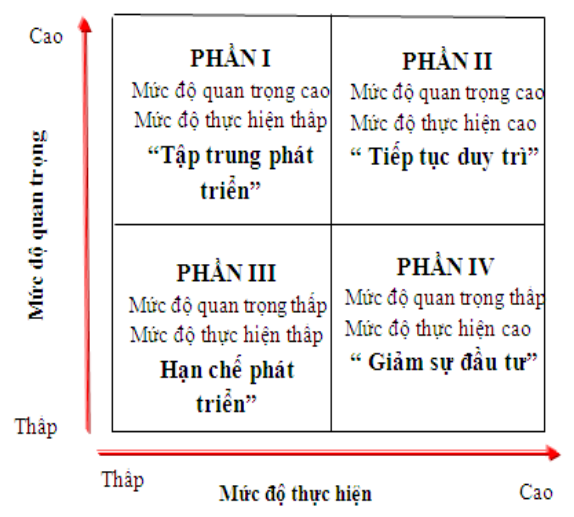

\section{Hình 1. Mô hình phân tích mức độ quan trọng và việc thực hiện (IPA)}

Phần I - Tập trung phát triển: Những thuộc tính nằm ở phần tư này được xem là rất quan trọng đối với khách hàng, nhưng mức độ thực hiện của nhà cung ứng dịch vụ rất kém. Kết quả này gợi ý cho nhà quản trị dịch vụ cung ứng chú ý đến những thuộc tính này, tập trung phát triển mức độ cung ứng dịch vụ nhằm thỏa mãn nhu cầu của khách hàng.

Phần II - Tiếp tục duy trì: Những thuộc tính ở phần tư này được xem là rất quan trọng đối với khách hàng, và nhà cung ứng dịch vụ cũng đã có mức độ thể hiện rất tốt. Nhà quản trị cung ứng dịch vụ nên tiếp tục duy trì và phát huy thế mạnh này.

Phần III - Hạn chế phát triển: Những thuộc tính nằm ở phần tư này được xem là có mức độ thể hiện thấp và không quan trọng đối với khách hàng. Nhà quản trị cung ứng dịch vụ nên hạn chế nguồn lực phát triển những thuộc tính này.

Phần IV - Giảm sự đầu tư: Những thuộc tính nằm ở phần tư này được xem là không quan trọng đối với khách hàng, nhưng mức độ thực hiện của nhà cung ứng rất tốt. Có thể xem sự đầu tư quá mức như hiện tại là vô ích. Nhà quản trị cung ứng dịch vụ nên sử dụng nguồn lực này tập trung phát triển những thuộc tính khác.

Mô hình IPA cho phép tổ chức xác định các thuộc tính hoặc sự phối hợp của chúng ảnh hưởng nhiều hay ít đến hành vi tiêu dùng của khách hàng. Đây được xem là thông tin có giá trị cho việc phát triển chiến lược Marketing trong tổ chức cũng như quá trình hình thành các công cụ quản lý hữu ích.

\section{Phương pháp nghiên cứu}

Nghiên cứu này thực hiện phương pháp nghiên cứu định tính và định lượng. Nghiên cứu định tính thông qua thảo luận nhóm 10 người bao gồm những sinh viên năm thứ 4 và một số cựu sinh viên đang công tác tại Trường. Nghiên cứu định tính nhằm xác định các thành phần và thang đo chất lượng dịch vụ giáo dục đại học phù hợp. Sau khi thực hiện nghiên cứu định tính đã khẳng định có 6 thành phần chất lượng dịch vụ giáo dục đại học: (1) Chương trình đào tạo (4 biến quan sát); (2) Kỹ năng giảng dạy của giảng viên (7 biến quan sát); (3) Cơ sở vật chất (5 biến quan sát); (4) Tương tác giữa nhà trường và doanh nghiệp (3 biến quan sát); (5) Hoạt động ngoại khóa (3 biến quan sát); (6) Chất lượng dịch vụ hỗ trợ cho sinh viên (3 biến quan sát). Thang đo ghi nhận đánh giá mức độ quan trọng sử dụng trong nghiên cứu này là dạng Likert 5 điểm (1- Rất không quan trọng, 5 - Rất quan trọng). Thang đo đánh giá mức độ thực hiện sử dụng trong nghiên cứu này là dạng Likert 5 điểm (1 - Rất không thỏa mãn, 5 - Rất thỏa mãn). Nghiên cứu định lượng với số mẫu sau khi sàng lọc là 193 mẫu. Nghiên cứu đã thực hiện kiểm định độ tin cậy thang đo sử dụng Cronbach's Alpha, phân tích nhân tố EFA, phân tích Paired Sample T-Test và phân tích theo mô hình IPA.

\section{Kết quả nghiên cứu và thảo luận}

\subsection{Kết quả nghiên cứu}

\section{Thống kê mẫu khảo sát}

Nghiên cứu này thực hiện phỏng vấn 2 lần, sau khi loại bỏ các bảng khảo sát cả hai lần, số mẫu chính thức là 193. Xét theo năm học, sinh viên năm thứ 1 có 38 sinh viên chiếm 19,7\%, năm thứ 2 có 58 sinh viên chiếm $30,1 \%$, năm thứ 3 có 50 sinh viên chiếm $25,9 \%$, năm thứ 4 
có 47 sinh viên chiếm 24,4\%. Xét theo khoa, Khoa Công nghệ thông tin 32 chiếm 16,6\%, Khoa Du lịch 14 (7,3\%), Khoa Kế toán 11 $(5,7 \%)$, Khoa Marketing 24 (12,4\%), Khoa Tài chính - Ngân hàng 58 (30,1\%), Khoa Quản trị kinh doanh 36 (18,7\%), Viện Đào tạo Sau đại học (học viên cao học) 18 chiếm 9,3\%. Xét theo giới tính, mẫu điều tra gồm 78 sinh viên nam, chiếm tỷ lệ 40,4\% và 115 sinh viên nữ, chiếm tỷ lệ 59,6\% (Bảng 4.3). Xét theo độ tuổi, mẫu điều tra gồm 122 sinh viên có độ tuổi từ 18-21, chiếm tỷ lệ $63,2 \%, 51$ sinh viên có độ tuổi từ 22 đến dưới 30 tuổi, chiếm tỷ lệ $26,4 \%, 20$ sinh viên có độ tuổi từ 31 đến dưới 45 tuổi, chiếm tỷ lệ $10,4 \%$.

\section{Kiểm định độ tin cậy thang đo bằng Cronbach's alpha}

Kết quả đo lường độ tin cậy bằng Cronbach's Alpha đều đạt yêu cầu. "Chương trình đào tạo" có hệ số Cronbach's Alpha $\alpha=0,825$; "Kỹ năng giảng dạy của giảng viên" $\alpha=0,888$; “Co sở vật chất" $\alpha=0,735$; "Tương tác giũa nhà trường và doanh nghiệp" $\alpha=0,831$; "Hoạt động ngoại khóa" $\alpha=0,838$; "Chất lương dịch vu hố trọ" sinh viên" $\alpha=0,837$.

\section{Phân tích mô hình IPA}

Theo kết quả nghiên cứu và đánh giá mức quan trọng của 6 chỉ tiêu và 25 tiêu chí, nhìn chung các tiêu chí thể hiện mức quan trọng đều có giá trị trung bình 3,66 - 4,15; không có giá trị thấp hơn điều này chứng tỏ rằng các tiêu chí đo lường đều quan trọng đối với sinh viên. Đối với việc đánh giá mức độ thực hiện, sinh viên đánh giá có giá trị trung bình $3,16-4,21$. Kết quả đánh giá P-I cho thấy sự khác biệt giá trị trung bình của từng cặp yếu tố và nhiều cặp yếu tố có p-value (Sig.) $<0,05$, cho biết chênh lệch về đánh giá của sinh viên với mức độ quan trọng và mức độ thực hiện của chất lượng giáo dục đại học tại trường Đại học Tài chính - Marketing đều có ý nghĩa với độ tin cậy 95\%.

Bảng 1. Đánh giá mức độ quan trọng và việc thực hiện của các thuộc tính chất lượng tại Trường Đại học Tài chính - Marketing

\begin{tabular}{|c|c|c|c|c|c|}
\hline TT & $\begin{array}{c}\text { Yếu tố chất lượng } \\
\text { dịch vụ giáo dục đại học }\end{array}$ & $\begin{array}{c}\text { Mức độ } \\
\text { quan trọng }\end{array}$ & $\begin{array}{l}\text { Mức độ } \\
\text { thực hiện }\end{array}$ & $\begin{array}{c}\text { Chênh } \\
\text { lệch } \\
\text { P-I }\end{array}$ & $\begin{array}{l}\text { Giá trị } \\
\quad P\end{array}$ \\
\hline \multicolumn{6}{|c|}{ CHƯƠNG TRÌNH ĐÀO TẠO } \\
\hline CTDT1 & Chương trình đào tạo có mục tiêu rõ ràng & 3,94 & 3,78 & $-0,16$ & 0,010 \\
\hline CTDT2 & $\begin{array}{l}\text { Chương trình đào tạo có chuẩn đầu ra rõ } \\
\text { ràng }\end{array}$ & 4,04 & 3,89 & $-0,15$ & 0,010 \\
\hline CTDT3 & $\begin{array}{l}\text { Nội dung chương trình đào tạo có kiến } \\
\text { thức kỹ năng đáp ứng nhu cầu thực tế }\end{array}$ & 4,01 & 3,75 & $-0,26$ & 0,001 \\
\hline CTDT4 & $\begin{array}{l}\text { Nội dung chương trình đào tạo giúp sinh } \\
\text { viên tìm công việc phù hợp }\end{array}$ & 3,90 & 3,75 & $-0,15$ & 0,028 \\
\hline \multicolumn{6}{|c|}{ KỸ NĂNG GIẢNG DẠY CỦA GIẢNG VIÊN } \\
\hline KNGD1 & Giảng viên giảng dạy rất dễ hiểu & 4,15 & 3,84 & $-0,31$ & 0,000 \\
\hline KNGD2 & $\begin{array}{l}\text { Giảng viên truyền đạt rõ ràng nội dung } \\
\text { giảng dạy }\end{array}$ & 4,15 & 3,85 & $-0,30$ & 0,000 \\
\hline KNGD3 & Giảng viên chuẩn bị bài giảng kỹ & 4,02 & 3,85 & $-0,17$ & 0,007 \\
\hline KNGD4 & $\begin{array}{l}\text { Giảng viên sử dụng hiệu quả thiết bị giảng } \\
\text { dạy }\end{array}$ & 4,14 & 3,90 & $-0,24$ & 0,000 \\
\hline
\end{tabular}




\begin{tabular}{|c|c|c|c|c|c|}
\hline TT & $\begin{array}{l}\text { Yếu tố chất lượng } \\
\text { dịch vụ giáo dục đại học }\end{array}$ & $\begin{array}{c}\text { Mức độ } \\
\text { quan trọng }\end{array}$ & $\begin{array}{c}\text { Mức độ } \\
\text { thực hiện }\end{array}$ & \begin{tabular}{|c|} 
Chênh \\
lệch \\
P-I
\end{tabular} & $\begin{array}{l}\text { Giá trị } \\
\quad \text { P }\end{array}$ \\
\hline KNGD5 & $\begin{array}{l}\text { Giảng viên khuyến khích sinh viên thảo } \\
\text { luận trong lớp }\end{array}$ & 4,05 & 4,16 & 0,11 & 0,123 \\
\hline KNGD6 & $\begin{array}{l}\text { Giảng viên tạo cơ hội cho sinh viên đặt } \\
\text { câu hỏi trong lớp }\end{array}$ & 3,95 & 3,79 & $-0,16$ & 0,017 \\
\hline KNGD7 & $\begin{array}{l}\text { Giảng viên khuyến khích sinh viên phát } \\
\text { biểu trong giờ học }\end{array}$ & 4,04 & 4,21 & 0,17 & 0,012 \\
\hline \multicolumn{6}{|c|}{ CƠ SỞ VẬT CHẤT } \\
\hline CSVC1 & $\begin{array}{l}\text { Phòng học sạch sẽ, rộng rãi và thông } \\
\text { thoáng }\end{array}$ & 3,84 & 3,55 & $-0,29$ & 0,000 \\
\hline CSVC2 & $\begin{array}{l}\text { Phương tiện giảng dạy (máy chiếu, micro } \\
\text {..) có thể áp dụng việc dạy học hiện đại }\end{array}$ & 4,10 & 3,84 & $-0,26$ & 0,000 \\
\hline CSVC3 & $\begin{array}{l}\text { Thư viện cung cấp đầy đủ tài liệu học tập } \\
\text { và nghiên cứu cho sinh viên }\end{array}$ & 3,95 & 3,26 & $-0,69$ & 0,000 \\
\hline CSVC4 & $\begin{array}{l}\text { Sinh viên có thể truy cập internet tại bất kỳ } \\
\text { đâu trong khuôn viên trường }\end{array}$ & 3,66 & 3,32 & $-0,34$ & 0,000 \\
\hline CSVC5 & $\begin{array}{l}\text { Cơ sở dữ liệu điện tử (tài liệu học tập, dữ } \\
\text { liệu nghiên cứu ...) phục vụ tốt cho việc } \\
\text { học tập và nghiên cứu }\end{array}$ & 3,87 & 3,38 & $-0,49$ & 0,000 \\
\hline \multicolumn{6}{|c|}{ TƯƠNG TÁC GIŨ’A NHÀ TRƯÒ̀NG VÀ DOANH NGHIỆP } \\
\hline NTDN1 & $\begin{array}{l}\text { Nhà trường tổ chức cho sinh viên những } \\
\text { chuyến đi thực tế tại doanh nghiệp }\end{array}$ & 3,83 & 3,17 & $-0,66$ & 0,000 \\
\hline NTDN2 & $\begin{array}{l}\text { Nhà trường mời diễn giả từ doanh nghiệp } \\
\text { trao đồi, chia sẻ tình hình thực tế tại doanh } \\
\text { nghiệp }\end{array}$ & 3,75 & 3,34 & $-0,41$ & 0,000 \\
\hline NTDN3 & $\begin{array}{l}\text { Nhà trường giới thiệu đơn vị thực tập cho } \\
\text { sinh viên }\end{array}$ & 4,11 & 3,24 & $-0,87$ & 0,000 \\
\hline \multicolumn{6}{|c|}{ HOẠT ĐỘNG NGOẠI KHÓA } \\
\hline HDNK1 & $\begin{array}{l}\text { Nhà trường tổ chức hoạt động ngoại khóa } \\
\text { cho sinh viên }\end{array}$ & 3,73 & 3,48 & $-0,25$ & 0,003 \\
\hline HDNK2 & $\begin{array}{l}\text { Sinh viên có đự̛̣c những kỹ năng sống } \\
\text { hữu ích từ hoạt động ngoại khóa }\end{array}$ & 3,69 & 3,36 & $-0,33$ & 0,000 \\
\hline HDNK3 & $\begin{array}{l}\text { Hoạt động ngoại khóa làm cho việc học } \\
\text { của sinh viên thú vị hơn }\end{array}$ & 3,84 & 3,34 & $-0,50$ & 0,000 \\
\hline \multicolumn{6}{|c|}{ CHẤT LỰ̛̣NG DỊCH VỤ HỖ TRỌ SINH VIÊN } \\
\hline CLDV1 & $\begin{array}{l}\text { Nhân viên khoa/phòng/ban làm việc khoa } \\
\text { học }\end{array}$ & 3,90 & 3,29 & $-0,61$ & 0,000 \\
\hline CLDV2 & $\begin{array}{l}\text { Nhân viên khoa/phòng/ban làm việc tận } \\
\text { tâm với sinh viên }\end{array}$ & 3,96 & 3,45 & $-0,51$ & 0,000 \\
\hline CLDV3 & $\begin{array}{l}\text { Nhân viên khoa/phòng/ban thân thiện với } \\
\text { sinh viên }\end{array}$ & 3,96 & 3,36 & $-0,60$ & 0,000 \\
\hline
\end{tabular}


Kết quả phân tích trên mô hình IPA thể hiện như sau:

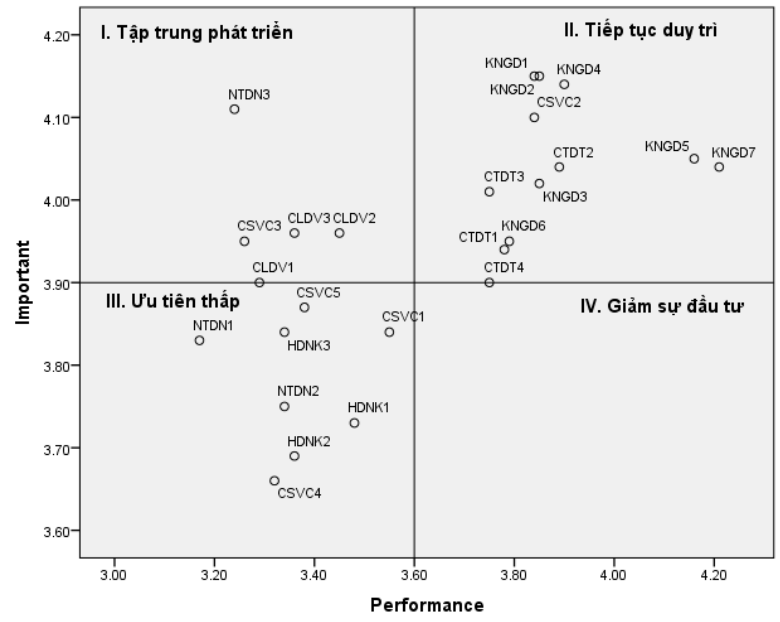

Nguồn: Dũ liệu phân tích của tác giả

\section{Hình 2. Mô hình mức độ quan trọng - mức độ thể hiện (IPA)}

Phần I - Tập trung phát triển: Thư viện cung cấp đầy đủ tài liệu học tập và nghiên cứu cho sinh viên (CSVC3); Nhà trường giới thiệu đơn vị thực tập cho sinh viên (NTDN3); Nhân viên khoa/phòng/ban làm việc khoa học (CLDV1); Nhân viên khoa/phòng/ban làm việc tận tâm với sinh viên (CLDV2); Nhân viên khoa/phòng/ban thân thiện với sinh viên (CLDV3). Các biến quan sát này có mức độ quan trọng cao nhưng mức độ thực hiện thì khá thấp, do đó đòi hỏi nhà trường phải chú trọng phát triển nhiều hơn.

Phần II - Tiếp tục duy trì: Chương trình đào tạo có mục tiêu rõ ràng (CTDT1); Chương trình đào tạo có chuẩn đầu ra rõ ràng (CTDT2); Nội dung chương trình đào tạo có kiến thức kỹ năng đáp ứng nhu cầu thực tế (CTDT3); Nội dung chương trình đào tạo giúp sinh viên tìm công việc phù hợp (CTDT4); Giảng viên giảng dạy rất dễ hiểu (KNGD1); Giảng viên truyền đạt rõ ràng nội dung giảng dạy (KNGD2); Giảng viên chuẩn bị bài giảng kỹ (KNGD3); Giảng viên sử dụng hiệu quả thiết bị giảng dạy (KNGD4); Giảng viên khuyến khích sinh viên thảo luận trong lớp (KNGD5); Giảng viên tạo cơ hội cho sinh viên đặt câu hỏi trong lớp (KNGD6); Giảng viên khuyến khích sinh viên phát biểu trong giờ học (KNGD7); Phương tiện giảng dạy (máy chiếu, micro ...) có thể áp dụng việc dạy học hiện đại (CSVC2). Các biến quan sát này có mức độ quan trọng cao và mức độ thực hiện cũng cao tương ứng. Vì thế trường cần tiếp tục duy trì và phát huy thế mạnh này.

Phần III - Uu tiên thấp: Phòng học sạch sẽ, rộng rãi và thông thoáng $(\mathrm{CSVC} 1)$; Sinh viên có thể truy cập internet tại bất kỳ đâu trong khuôn viên trường (CSVC4); Cơ sở dữ liệu điện tử (tài liệu học tập, dữ liệu nghiên cứu ...) phục vụ tốt cho việc học tập và nghiên cứu (CSVC5); Nhà trường tổ chức cho sinh viên những chuyến đi thực tế tại doanh nghiệp (NTDN1); Nhà trường mời diễn giả từ doanh nghiệp trao đổi, chia sẻ tình hình thực tế tại doanh nghiệp (NTDN2); Nhà trường tổ chức hoạt động ngoại khóa cho sinh viên (HDNK1); Sinh viên có được những kỹ năng sống hữu ích từ hoạt động ngoại khóa (HDNK2); Hoạt động ngoại khóa làm cho việc học của sinh viên thú vị hơn (HDNK3). Như chúng ta đã thấy trên cơ sở phân tích mức độ quan trọng và mức độ thực hiện, ma trận IPA được xây dựng cho mức độ thực hiện chất lượng dịch vụ giáo dục tại trường Đại học Tài chính - Marketing. Có 8 yếu tố rơi vào khu vực "Uu tiên thấp" theo các đối tượng khảo sát, các yếu tố này được đánh giá không cao về mặt thực hiện và cũng ít quan trọng hơn so với các yếu tố khác. Các biến quan sát này đa phần không đóng vai trò quá quan trọng với sinh viên và mức độ thực hiện tại các yếu tố này cũng khá thấp nên nhà trường cũng không cần quá chú trọng phát triển nhiều. Chính vì thế nhà trường nên tập trung phát triển những thuộc tính khác.

Phần IV - Hạn chế đầu tư: Các biến quan sát này có mức độ quan trọng thấp nhưng mức độ thực hiện tương đối cao. Tuy nhiên không có yếu tố nào rơi vào khu vực này. 


\subsection{Thảo luận kết quả nghiên cứu}

Kết quả phân tích từ mô hình IPA cho thấy các thuộc tính trong 6 yếu tố cấu thành chất lượng dịch vụ giáo dục đại học tại trường Đại học Tài chính - Marketing thông qua khảo sát sinh viên được phân tán trong 3 phần I, II, III của mô hình IPA, không có thuộc tính nào rơi vào phần IV. Giảm đầu tư. Kết quả này đã phản ánh được đúng thực tiễn ở trường Đại học Tài chính - Marketing hiện nay. Cách đo lường chất lượng dịch vụ giáo dục đại học dưới góc nhìn của sinh viên theo mô hình IPA chi tiết rõ ràng và đúng thực tiễn hơn các mô hình nghiên cứu trước. Nghiên cứu này so với nhiều nghiên cứu trước có sự khác biệt có ý nghĩa khoa học và thực tiễn. Hầu hết các nghiên cứu trước đây ở Việt Nam khi thực hiện đánh giá về chất lượng dịch vụ thường áp dụng thang đo SERVQUAL của Parasuraman. Tuy nhiên, chất lượng dịch vụ là một khái niệm phức tạp và vì vậy việc tiếp cận đánh giá nó trên những góc độ khác nhau lại có những đóng góp về mặt thực tiễn quan trọng. Kết quả nghiên cứu mô hình IPA lại cho các nhà quản lý có được cái nhìn mang tính “trực diện” hơn đối với chất lượng dịch vụ.

\section{Kết luận và hàm ý quản trị}

\subsection{Kết luận}

Trên nền tảng lý thuyết chất lượng dịch vụ giáo dục đại học dưới góc nhìn sinh viên, nghiên cứu này đã xác định 6 yếu tố thành phần chất lượng dịch vụ giáo dục đại học gồm Chương trình đào tạo, Kỹ năng giảng dạy của giảng viên, Cơ sở vật chất, Tương tác giữa nhà trường và doanh nghiệp, Hoạt động ngoại khóa, Chất lượng dịch vụ hỗ trợ sinh viên. Nghiên cứu đã thực hiện khảo sát 193 mẫu và đo lường độ tin cậy thang đo bằng Cronbach Alpha, kiểm định sự khác biệt Paired Sample T-test và phân tích theo mô hình IPA. Kết quả nghiên cứu chất lượng dịch vụ giáo dục đại học cho thấy: 5 thuộc tính chất lượng nằm Phần I, 12 thuộc tính nằm Phần $\mathrm{II}, 8$ thuộc tính nằm Phần $\mathrm{III}$, không có thuộc tính nằm $\mathrm{Phần} \mathrm{IV.}$

\subsection{Hàm ý quản trị}

\subsubsection{Chuoong trình đào tạo}

Theo kết quả phân tích mô hình IPA thì các biến quan sát của chương trình đào tạo đều ở phần II (tiếp tục duy trì). Giá trị trung bình mức độ thực hiện đều thấp hơn tầm quan trong P-I đều nhỏ hơn không. Do vậy, Trường cần quan tâm nhiều hơn đến chương trình đào tạo gồm: (1) Cần rà soát đảm bảo chương trình đào tạo có mục tiêu rõ ràng, có chuẩn đầu ra nội dung chương trình đào tạo phải có kiến thức, kỹ năng đáp ứng nhu cầu thực tế và phù hợp với công việc sau tốt nghiệp; (2) Chương trình đào tạo cần phải được xây dựng có sự tham gia của người sử dụng lao động và dự báo được thị trường lao động trong tương lai.

\subsubsection{Kỹy năng giảng dạy của giảng viên}

Theo kết quả phân tích mô hình IPA các biến quan sát kỹ năng giảng dạy nằm ở phần II (tiếp tục duy trì). Trong đó có một biến sinh viên đánh giá $\mathrm{P}-\mathrm{I}>0$ là Giảng viên khuyến khích sinh viên thảo luận trong lớp. Như vậy có thể nhận xét rằng trong quá trình giảng dạy, giảng viên đã cho sinh viên thảo luận nhiều nhưng sinh viên cho rằng không quan trọng. Điều này phản ánh được tâm lý chung của người đi học. Các biến quan sát khác $\mathrm{P}-\mathrm{I}<0$. Như vậy, việc nâng cao chất lượng đội ngũ giảng viên là cần thiết. Để nâng cao kỹ năng giảng dạy, bài báo đề xuất một số hàm ý quản trị sau: (1) Nhà trường khuyến khích giảng viên phát huy tính chủ động, tích cực, tự giác trong tự học, tự rèn nâng cao năng lực giảng dạy của đội ngũ giảng viên; (2) Đảm bảo tuyển dụng và xây dựng được đội ngũ giảng viên có chất lượng cao; (4) Xây dựng kế hoạch đào tạo, bồi dưỡng giảng viên phải được xây dựng một cách chi tiết, cụ thể phù hợp với ngành, chuyên ngành của từng 
giảng viên hiện tại. (5) Đổi mới công tác nghiên cứu khoa học trong đội ngũ giảng viên; (7) Tạo cơ hội cho cán bộ, giảng viên đi học tập, trao đổi với các trường đại học trong nước và nước ngoài, thông qua đó giúp cán bộ, giảng viên tiếp cận những quan điểm giáo dục hiện đại, những thành tựu mới về khoa học, công nghệ cũng như các phương pháp giảng dạy tiên tiến để có thể áp dụng vào thực tiễn nhà trường, từng bước nâng cao vị thế và uy tín của Trường, đáp ứng yêu cầu của công tác đào tạo trong giai đoạn hiện nay.

\subsubsection{Co sở vật chất}

Để cải tiến cơ sở vật chất, Trường cần tập trung cải tiến một số nội dung như: phòng học, phương tiện giảng dạy (máy chiếu, micro ...), tài liệu học tập và nghiên cứu cho sinh viên tại thư viện, mạng internet, và cơ sở dữ liệu điện tử phục vụ cho sinh viên nghiên cứu và học tập để thực hiện được, bài báo đưa ra một số hàm ý quản trị như sau: (1) Cải tiến hệ thống công nghệ thông tin của Trường; (2) Cần chiến lược về cơ sở hạ tầng dài hạn để tiến đến không thuê mướn trụ sở; (3) Đẩy mạnh đầu tư thư viện điện tử và số hóa các tài liệu học tập.

\subsubsection{Tương tác giũa nhà truoòng và doanh} nghiệp

Hợp tác giữa nhà trường và doanh nghiệp là sự hợp tác giữa hai bên để thực hiện các mục đích, chương trình đào tạo đã được hai bên xác lập. Một số hàm ý quản trị: (1) Trong mối quan hệ hợp tác này, nhà trường đóng vai trò là đơn vị chủ trì, chịu trách nhiệm chính trong đào tạo như thực hiện nội dung, chương trình, chất lượng đào tạo, cấp chứng nhận, bằng cấp đào tạo cho người được đào tạo; (2) Doanh nghiệp đóng vai trò là đơn vị phối hợp, hỗ trợ, chịu trách nhiệm về tổ chức, quản lý, phục vụ cho quá trình đào tạo, sử dụng sản phẩm đào tạo; (3) Nâng cao vai trò của bộ phận hợp tác doanh nghiệp là rất quan trọng; (4) Sự tham gia của tất cả cán bộ quản lý, giảng viên, cựu sinh viên để có thông tin quan trọng, nắm bắt nhanh chóng nhu cầu cấp thiết của doanh nghiệp.

\subsubsection{Hoạt động ngoại khóa}

Theo mô hình phân tích IPA các biến quan sát hoạt động ngoại khóa rơi vào khu vực “Uu tiên thấp". Mặc dù, sinh viên đánh giá ít quan trọng nhưng cũng đánh giá không cao về mặt thực hiện do vậy, bài báo đề xuất một số hàm ý quản trị sau: (1) Cần xây dựng cách đánh giá rèn luyện rõ ràng để khuyến khích sinh viên tham gia các hoạt động ngoại khóa; (2) Đội ngũ giảng viên, viên chức có kiến thức, kỹ năng tổ chức các hoạt động ngoại khóa hấp dẫn; (3) Xây dựng kế hoạch hoạt động ngoại khóa một cách chi tiết, cụ thể, thông qua kế hoạch năm học và của từng khóa, từng đối tượng và gắn với hoạt động kỷ niệm những ngày lễ lớn của dân tộc, nhà trường, đơn vị.

\subsubsection{Chất luợng dịch vụ hỗ trợ sinh viên}

Qua phân tích mô hình IPA yếu tố chất lượng dịch vụ hỗ trợ sinh viên nằm ở phần $\mathrm{I}$ - Tập trung phát triển. Do đó, để cải tiến chất lượng dịch vụ hỗ trợ sinh viên thông qua cách làm việc của nhân viên đối với sinh viên, bài báo đề xuất một số hàm ý quản trị: (1) Trường cần rà soát, xây dựng quy trình làm việc khoa học cho nhân viên nhằm phục vụ sinh viên một cách tốt nhất; (2) Xây dựng hệ thống khảo sát lấy ý kiến của sinh viên thường xuyên; (3) Nâng cao kỹ năng thực hiện công việc cho nhân viên; (4) Xây dựng văn hóa lấy sinh viên làm trung tâm.

\subsection{Hạn chế và hướng nghiên cứu tiếp theo}

Bài báo này chỉ đề xuất mô hình 6 yếu tố cấu thành nên chất lượng dịch vụ giáo dục đại học. Nhiều nghiên cứu trước cũng chỉ ra còn nhiều yếu tố thành phần khác. Do vậy, nghiên cứu này có thể mở rộng phân tích thêm các thành phần khác. 


\section{TÀI LIẸU THAM KHẢO}

\section{Tiếng Việt}

Huỳnh Văn Thái. (2017). Vận dụng mô hình IPA để đánh giá chất lượng dịch vụ đào tạo tại Trường Cao đẳng Công nghiệp Tuy Hòa. Tạp chí Khoa họ - Trường Đại học Đồng Tháp.

Phạm Thị Ly. (2013, November 30). Về quan hệ hợp tác giũua nhà truờng và doanh nghiệp. Retrieved from Ly Pham: http://lypham.net

\section{Tiếng Anh}

Cheng, Y.C and Tam, W.M. (1997). Multi-models of quality in education. Quality Assurance in Education, $22-51$

Dirr, P. (2014, August 12). Putting Principles into Practice: Promoting effective support services for students in distance learning programs. Retrieved from http://peterdirr.freehostia.com

Firdaus, A. (2006). The development of HEdPERF: A new measuring instrumant of service quality for the higher education sector. International Journal of Consumer Studies, 569-581.

Harvey, L., and Green, D. (1993). Defining quality. Assessment and Education in Higher Education, 9-34.

Jain, R. \&. (2013). Developing a scal to measure students' perception of service quality in the Inđian context. The TQM Journal, 276-294.

Joseph, M. and Joseph, B. (1997). Service quality in education: a student perspective. Quality Assurance in Education, 15-21.

Kyriacou, C. (2007). Essential teaching skills, 3rd Ed. United Kingdom: Nelson Thornes Ltd.

Lagrosen, S. e. (2004). Examination of the dimentions of qua lity in higher education. Quality Assurance in Education, 61-69.

Martilla J.A. \& James J.C. . (1977). Important Performance Analysis. Journal of Marketing, 77-79.

NAAC. (2008). Best practice series: Curricular aspects - Case presentations. Bangalore, India: Shakthi Printech.

O'Neil and Palmer. (2004). Importance - Performance Analysis: A useful tool for directing continous quality improvement in higher education. Quality Assurance in Education, 39-52.

Parasuraman, A. \&. (1985). A conceptual model of service quality and ít implications for future research. Journal of Marketing, 41-50.

Peng, P.J. and Samah, A.J.A. (2006). Measuring students' satisfaction for quality education in a e-learning university. Unitar E-Journal, 11-21.

Print, M. (1987). Curiculum Development and Design. Sydney: Allen \& Unwin.

Silva $\&$ et al. (2017). Measurement of perceived service quality in higher education institutions: A review of HEdPERF scale use. Quality Assurance in Education, 415-439.

Simpson, O. (2002). Supporting Students in Online, Open and Distance Learning. London: Kogan Page Limited.

Sumaedi, S. \&. (2012). An empirical study of state university students' perceived service quality. Quality Assurance in Education, 164-183.

Tsinidou, M \& et al. (2010). Evaluation of the factors that determine quality in higher education: An empirical study. Quality Assurance in Education, 227-244.

Vijaya Sunder, M. (2015). Constructs of quality in higher education quality. International Journal of Productivity and Performance Management, 1091-1111.

Wragg, E. (2005). The art and science of teaching and learning: The selected works of Ted Wragg. London: Routledge Falmer. 